\title{
Relation between vitamin D deficiency and osteoarthritis
}

\section{Gharaibeh $A^{* 1}$, Kupershtein $\mathbf{M}^{1}$, Gharaibeh $\mathbf{M}^{2}$, Filip $\mathbf{V}^{1}$, Sokol ${ }^{1}$, Lackova $\mathrm{A}^{3}$, Folvarsky $\mathbf{M}^{1}$, Beres $\mathbf{M}^{1}$, Mitro $\mathrm{I}^{1}$, Ivanecky $\mathrm{S}^{4}$, Habinakova $\mathbf{M}^{5}$, Naser $\mathbf{Y}^{1}$, Cellar $\mathbf{R}^{1}$, Schreierova $\mathrm{D}^{1}$ and Lacko $\mathbf{M}^{6}$}

${ }^{1}$ Orthopaedic Surgeon, Department of Orthopaedics and musculoskeletal trauma, UNLP, Slovakia

${ }^{2}$ Teaching Department of dentistry, Princes Basma Teaching Hospital, Consultant of oral\& maxillofacial Surgery, Jordan

${ }^{3}$ Neurologist, Department of Neurology, UNLP, Slovakia

${ }^{4}$ Trauma Surgeon, Teaching Department of trauma, UNLP, Slovaka

${ }^{5}$ Department of Medical Informatics, Faculty of Medicine, UPJŠ, Slovakia

${ }^{6}$ Head of Teaching Department of Orthopaedics and musculoskeletal trauma, UNLP, Slovakia

\section{Research Article \\ Volume 4 Issue 1}

Received Date: May 20, 2020

Published Date: July 02, 2020

DOI: $10.23880 /$ jobd-16000194

*Corresponding author: Ahmad Gharaibeh, MPH, PhD, Assistant professor, Orthopaedic Surgeon, teaching Department of Orthopaedics and musculoskeletal trauma, UPJS, UNLP, Kosice, Slovakia, Tel: +421948612693; Email: gharaibeh@seznam.cz

\section{Abstract}

Purpose of the Work: The main objective of our work is to determine the relationship between osteoarthritis and deficiency of vitamin D in our region in central Europe and to make a database for further researches, to be effective in investigating, controlling, and preventing $\mathrm{OA}$ and vitamin D deficiency in our population.

Methodology: We perform a retrospective study in adult patients $\geq 25$ years with osteoarthritis big joints from their health records, which were seen at the osteology clinic of University Hospital Louise Pasteur during the year 2018. The authors analyse the blood tests of Vitamin D level, calcium level, phosphorus level, B ALP, glomerular filtration rate (GFR) levels in the serum of these patients.

Results: There were 47 patients with osteoarthritis. All of these patients had Vitamin D deficiency. The mean age of our group is 71 years. $12.8 \%$ (7) are male and $87.2 \%$ (41)are female. The mean of vitamin D is $24 \mathrm{mmol} / \mathrm{l}$ (normal range $75-200 \mathrm{nmol} / \mathrm{l}$ ), Calcium level with in normal range, Phosphorus level mean was within normal range and B ALP level within normal.

Conclusion: Osteoarthritis increased by deficiency of vitamin D level in blood serum and increasing with age. Vitamin D is a fat-soluble vitamin that regulates calcium and phosphorus metabolism, maintenance of the normal skeletal and muscular systems. Most of the patients show OA in hip and knee and in advanced age 71 years. Vitamin D supplementation may be a safe method to treat and prevent $\mathrm{OA}$.

Keywords: Vitamin D, osteoarthritis, deficiency, relation 


\section{Journal of Orthopedics \& Bone Disorders}

\section{Introduction}

Vitamin D is a fat-soluble vitamin that regulates calcium and phosphorus metabolism, which controls the growth, development, and maintenance of the normal skeletal and muscular systems. Low levels of vitamin d concentration are associated with the incidence of cancer, autoimmune diseases, infectious diseases, and cardiovascular diseases. The incidence of people with vitamin d deficiency tends to increase as age increase. In humans, serum 250 hd is the main diagnostic value; it reflects the vitamin $d$ concentration that is derived from dietary absorption and skin synthesis. Vitamin d deficiency and insufficiency are increasingly serious global problems [1].

Osteoarthritis is one of the most common joint disorders worldwide and its incidence is increasing with age and risk factors, mainly obesity and sedentary lifestyle. Risk factors of OA can be modifiable like diet, daily activity and smoking and non-modifiable like genetics, gender and age. Risk factors can be joint level factors as injury, malalignment and abnormal loading of the joints. OA is the 11th cause of disability in the world. This is the leading cause of activity limitations, particularly walking, and affects participation and quality of life [2-4]. Patients with $\mathrm{OA}$ are at greater risk of all-cause mortality, particularly for cardiovascular diseases than the general population. It has importance in early diagnosis and correct management of disease progression using medication and surgical therapy $[2,5]$. The decrease in calcium and phosphate absorption from the intestine which leads to abnormal bone metabolism [6].

In patients with knee osteoarthritis according to Kellgren and Lawrence radiographic grading scale the pain decrease with vitamin $\mathrm{D}$ deficiency and decrease dysfunction and a poorer quality of life in 100 patients in Turkey [1].

\section{Material and Methods}

We perform a retrospective study in adult patients $\geq 25$ years with osteoarthritis from their health records, which were seen at the osteology clinic of University Hospital Louise Pasteur during the year 2018. The authors analyse the blood tests of Vitamin D level, calcium level, phosphorus level, B ALP, glomerular filtration rate (GFR) levels in the serum of these patients. This study was approved in accordance with the ethical standards of university hospital committee on human experimentation, and the informed consent of all patients was obtained.

\section{Purpose of the Work}

The main objective of our work is to determine the effective of vitamin D deficiency on patients with big joints osteoarthritis.

\section{Study Location}

Osteology clinic in University Hospital Louise Pasteur with 1356 beds is the second largest Slovak university hospital and provides consultation health care to patients not only from the Kosice city area but form the whole Slovakia.

\section{Study Population}

All patients with vitamin D deficiency and osteoarthritis in our University Hospital (osteology clinic in University Hospital Louise Pasteur Rastislavova 43).

\section{Study Period}

From 1 January 2018 to 31 December 2018.

\section{Data Collection}

The data will be collected from the UNLP osteology clinic records.

\section{Data Analysis}

Collected data will be analysed by excel program.

\section{Results}

There were 47 patients with osteoarthritis. All of these patients had Vitamin D deficiency related to osteoarthritis. The mean age of our group is 71 years (51years and highest 86 years old) (Table 1 ).

\begin{tabular}{|c|c|}
\hline \multicolumn{2}{|c|}{ Age } \\
\hline Mean & 71.0212766 \\
\hline Standard Error & 1.077260273 \\
\hline Median & 71 \\
\hline Mode & 75 \\
\hline Standard Deviation & 7.385324348 \\
\hline Sample Variance & 54.54301573 \\
\hline Kurtosis & 0.109487666 \\
\hline Skewness & -0.334316303 \\
\hline Range & 35 \\
\hline Minimum & 51 \\
\hline Maximum & 86 \\
\hline Sum & 3338 \\
\hline Count & 47 \\
\hline
\end{tabular}

Table 1: Age statistic data.

Female were 41 patients and 6 male patients (Graph 1). 


\section{Journal of Orthopedics \& Bone Disorders}

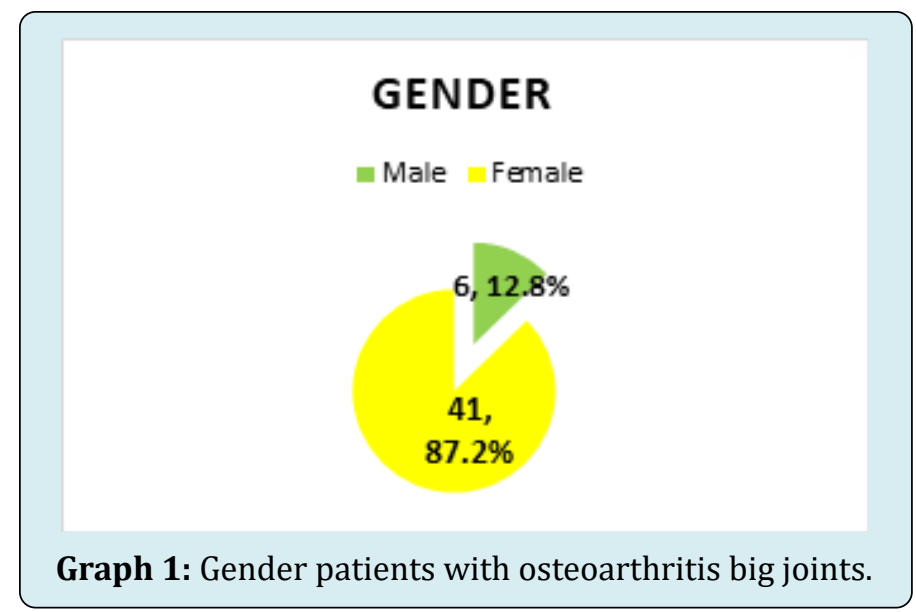

Pearson's correlation coefficient $(r=-0.16)$ was used to test the relationship between vitamin $\mathrm{D}$ and patient age. This showed that there is a slight negative relationship between vitamin $D$ values and patient age (the higher the patient's age, the lower vitamin $\mathrm{D}$ values), but a statistically insignificant relationship ( $p=0.282)$. There is no statistically significant relationship between vitamin $\mathrm{D}$ and other variables analysed (Table 2).

Between Vitamin D and the patient's age a Pearson correlation coefficient $(\mathrm{r}=-0.16)$ was used to test the relationship between them. It shows that there is a slight negative relationship between them (the higher the patient's age, the lower the vitamin $D$ value) but the $p$ value is insignificant relationship $(p=0.282)$. A statistically significant relationship is not even between vitamin $D$ and other analysed variables (Graph 2).

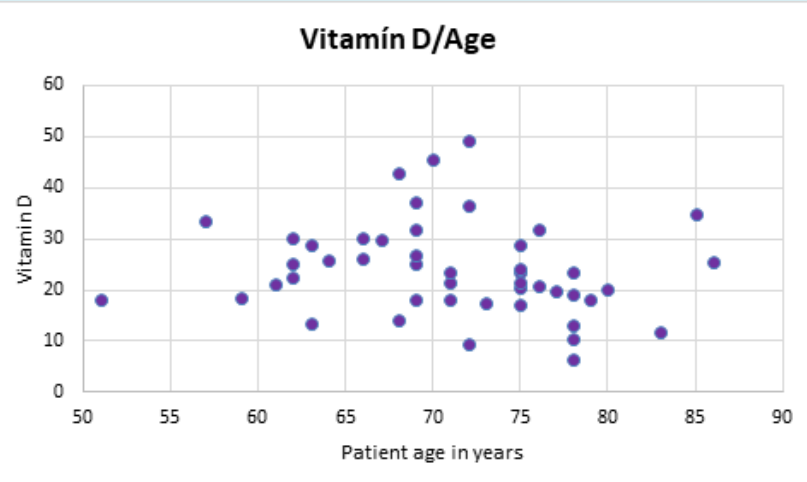

Graph 2: The relation between Vitamin D level in blood serum and the age of patient.

The normal range of vitamin D levels is $75-200 \mathrm{mmol} / \mathrm{l}$, the mean value represents the average number of vitamin $D$ that the patients in our study have in their results and it is accounts for 24.099 .

\begin{tabular}{|c|c|}
\hline \multicolumn{2}{|c|}{ Vitamin D3 } \\
\hline Mean & 24.0993617 \\
\hline Standard Error & 1.334137507 \\
\hline Median & 23.44 \\
\hline Mode & 18.26 \\
\hline Standard Deviation & 9.146385935 \\
\hline Sample Variance & 83.65637567 \\
\hline Kurtosis & 0.645623935 \\
\hline Skewness & 0.66414838 \\
\hline Range & 43.02 \\
\hline Minimum & 6.39 \\
\hline Maximum & 49.41 \\
\hline Sum & 1132.67 \\
\hline Count & 47 \\
\hline
\end{tabular}

Table 2: Vitamin D levels statistics.

The relation between Vitamin $D$ serum level and the values of Calcium, phosphorus, B ALP, GFR serum level is not significant and within normal range (Table 3 ).

\begin{tabular}{|c|c|c|c|c|c|}
\hline & & Calcium & Phosphorus & B ALP & GFR \\
Vitamin & $\mathbf{r}$ & -0.095 & 0.16 & 0.077 & 0.255 \\
D & $\mathbf{p}$ & 0.527 & 0.283 & 0.624 & 0.139 \\
& $\mathbf{N}$ & 47 & 47 & 43 & 35 \\
\hline
\end{tabular}

Table 3: Correlation between the blood tests.

\section{Discussion}

This study confirmed that vitamin D deficiency (serum $25(\mathrm{OH}) \mathrm{D}<15 \mu \mathrm{g} / \mathrm{L})$ cause osteoarthritis. We collect 47 patients with osteoarthritis and measure their vitamin D levels, Calcium, phosphorus, GFP and B ALP. We saw that every one of our patients have a deficiency of vitamin $D$ and most of them were old age women (average age 71 years).

In Garfinkel review study, which was done about detection the effect of vitamin $D$ on articular cartilage and the development of $\mathrm{OA}$, is shown results of vitamin $\mathrm{D}$ that plays a role in articular cartilage degeneration and poses a risk factor for OA [7] the study group in Slovakia confirmed this fact.

A research in Boston by Fang tries to check the association of Vitamin D deficiency with the progression of knee osteoarthritis; their results were that individuals deficient in vitamin $\mathrm{D}$ have an increased risk of knee osteoarthritis progression [8], which contribute and match our result that vitamin $\mathrm{D}$ deficiency relates 


\section{Journal of Orthopedics \& Bone Disorders}

to osteoarthritis and have important role in developing osteoarthritis disease.

\section{Conclusion}

Osteoarthritis is one of the most common joint disorders worldwide increased by deficiency of vitamin $\mathrm{D}$ level in blood serum and its incidence is increasing with age and risk factors. Vitamin D is a fat-soluble vitamin that regulates calcium and phosphorus metabolism, maintenance of the normal skeletal and muscular systems. Most of the patients show OA in hip and knee and in advanced age 71 years.

Vitamin D supplementation may be a safe method to treat and prevent $\mathrm{OA}$, but future research is required to settle the debate as to the role of vitamin D deficiency in the development and progression of OA.

Conflict of Interest Statement: The author declared that the research was conducted in the absence of any commercial or financial relationship that could be construed as a potential conflict of interest.

Limitation of Study: The number of patients was small and most of them were female sex gender. Also, due to the type of study, we did not follow up on the patients' OA progressions and vitamin D levels.

\section{Acknowledgment}

The authors thank all the staff of Osteology clinic for their kind support.

Funding: The work was supported by the Scientific Grant Agency of the Ministry of Education, Science, Research and Sports of the Slovak Republic - VEGA no.1/0598/20.

\section{References}

1. Alkan G, Akgol G (2017) Do vitamin D levels affect the clinical prognoses of patients with knee osteoarthritis? J Back Musculoskelet Rehabil 30(4): 897-901.

2. Gharaibeh A, Sepitka R, Stolfa S, Al Wadiya A, Spakova T, et al. (2020) 'Treatment of knee osteoarthritis with intra-articular application of autologous plasma rich in platelets - long term follow up. Wulfenia 27(2): 156-188.

3. Gharaibeh A, Al Wadiya A, Stolfa S, Sepitka R, Lacko M, et al. (2016) Comparison of Side effects and the need of pain killers in the treatment of knee osteoarthritis by PlasmaRich Platelets, Hyaluronic acid and Corticosteroids. Slov Chir 13(3-4): 72-75.

4. Gharaibeh A, Lacko M, Stolfa S, Tothova T, Rosocha J, et al. (2014) Plasma rich in platelets versus hyaluronic acid and corticosteroids in the treatment of knee osteoarthritis. Slov Chir 11(3): 92-96.

5. Spakova T, Rosocha J, Lacko M, Harvanova D, Gharaibeh A (2012) Treatment of knee joint osteoarthritis with autologous platelet-rich plasma in comparison with hyaluronic acid. American Journal of Physical Medicine \& Rehabilitation 91(5): 411-417.

6. Gani LU, How How C (2015) Vitamin D deficiency. Singapore medical journal 56(8): 433-437.

7. Garfinkel R, Dilisio M, Agrawal D (2017) Vitamin D and Its Effects on Articular Cartilage and Osteoarthritis Orthop J Sports Med 5(6): 2325967117711376.

8. Fang Fang Zhang, Jeffrey B Driban, Grace H Lo, Lori Lyn Price, Sarah Booth, et al. (2014) Vitamin D Deficiency Is Associated with Progression of Knee Osteoarthritis J Nutr 144(12): 2002-2008.

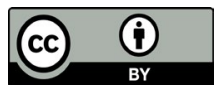

